\title{
Desarrollo humano
}

\section{y convergencia en economías} de aglomeración:

\author{
un debate entre Amartya Sen y Paul Krugman*
}

\section{Human development and convergence in agglomeration economies: \\ A debate between Amartya Sen and Paul Krugman}

David Ricardo Racero Mayorca**

Recibido: 15 de octubre de 2014

Revisado: 22 de enero de 2015

Aprobado: 16 de marzo de 2015

\section{Resumen}

El presente trabajo plantea un diálogo entre dos de los premios Nobel de Economía más influyentes de las últimas décadas: Paul Krugman y Amartya Sen. La tesis central del trabajo consiste en plantear la necesidad de acudir a la propuesta de política económica adelantada por Sen, referida a "bienes públicos", para poder generar condiciones de desarrollo no segregacionistas en economías de aglomeración. El presente documento

* El presente trabajo es una versión adaptada de la investigación presentada por el autor para optar al título de magíster en Ciencias Económicas en la Universidad Nacional de Colombia (2012). Como citar: Racero, D. (2014). Desarrollo Humano y Convergencia en economías de aglomeración. Revista CIFE, 16(25), 43-70.

** Filósofo y Magister en Ciencias Económicas, Universidad Nacional de Colombia Subdirector para la Juventud Secretaría Distrital de Integración Social, Correo: drracerom@gmail.com 
evidencia que el proceso que Krugman describe sobre el crecimiento de las economías de aglomeración deja inevitablemente zonas rezagadas que, por no estar cerca de los centros industriales y de producción, quedan suspendidas en la pobreza. De esta manera, la noción de "desarrollo" más amplia que utiliza Sen hace posible acudir a la generación de mecanismos progresivos que permitan disminuir tal desigualdad mediante políticas distributivas para la ampliación de capacidades. El diálogo conceptual de los dos autores nos posibilita la construcción de un modelo hipotético que tiene como centro la maximización de la utilidad por parte de los hogares (aumento de capacidades en sentido de Sen) en un contexto de aglomeración en perspectiva de Krugman. Al final se realiza una proyección dinámica del modelo mediante la plataforma de simulación Netlogo para soportar la propuesta conceptual.

Palabras clave: aglomeración, desarrollo humano, geografía económica, aumento de capacidades, desigualdad, distribución.

Clasificación JEL: D63, H41, I3, O15

\begin{abstract}
This paper presents a dialogue between two of the most influential Nobel Economics prize in recent decades: Paul Krugman and Amartya Sen. The central thesis of the job is to raise the need to resort to economic policy proposal advanced by Sen referred to "public goods" to create conditions for development no segregationist in agglomeration economies. This paper shows that the process described Krugman on the growth of agglomeration economies that inevitably leaves stragglers not be near the industrial centers and production are suspended in poverty areas. Thus, the broader notion of "development" using Sen lets go to the creation of progressive mechanisms to lessen such inequality by distributive capacity for expansion policies. The conceptual dialogue between the two authors allows us to build a hypothetical model that has at its center the utility maximization by households (capacity building in the sense of Sen) in a context of agglomeration in perspective Krugman. Ultimately, a dynamic model projection is performed using the Netlogo simulation platform to support the conceptual proposal.
\end{abstract}

Keywords: Agglomeration, human development, economic geography, capacity building, inequality distribution.

Classification JEL: D63, H41, I3, O15 


\section{Introducción}

Dentro del pensamiento económico de las últimas dos décadas, se pueden reconocer las teorías de Paul Krugman y de Amartya Sen como corrientes conceptuales de la disciplina que renuevan el concepto de desarrollo económico. Krugman hace toda una reconstrucción histórica y crítica de lo que denomina "teoría del alto desarrollo" post Segunda Guerra Mundial, retomando postulados importantes y válidos que fueron olvidados por "su incapacidad de ser modelados", que llegan, junto con la geografía económica del momento, a ser elementos fundamentales de su teoría. (Krugman, 1995).

Por su parte, es conocido de manera universal el debate que ha sostenido Sen con respecto al entendimiento de la noción de "desarrollo", así como su aporte significativo del concepto de "desarrollo humano como libertad" y aumento de "capacidades" como componentes centrales de este (ver, por ejemplo, Sen 1999 y 1998).

Sin embargo, el hecho de que los dos autores sustenten parte de sus teorías en la tradición del desarrollo económico no significa que sus estructuras conceptuales se apropien de la misma manera de la noción de desarrollo ni que compartan al unísono la forma como comprenden su proceso en las sociedades. De ahí sus énfasis diferentes: en la ubicación geográfica de la economía, por un lado, y en la preocupación por la inversión social como posibilidad de desarrollo, por el otro.

El presente trabajo hace un seguimiento de las concepciones comunes y diversas que pueden tener las dos teorías conforme a las ideas y a los conceptos que manejan de la noción de "desarrollo". Con ello se plantea una discusión analítica que invita a preguntar por un posible acercamiento de los autores desde ciertos componentes de sus visiones particulares. La metodología de investigación comparada que se propone en el presente escrito permite hacer una lectura de la teoría descriptiva que explica el desarrollo económico desde un enfoque espacial (Krugman), a la luz de las preocupaciones y propuestas de una teoría normativa que plantea la acción de la política económica en el intento de superar la desigualdad con acciones afirmativas de distribución (Sen). Teniendo presente los procesos de concentración geográfica que Krugman explica a partir de la ubicación de empresas, se pregunta por la necesidad de mejorar las condiciones de vida de las personas (hogares), de tal manera que se pueda generar un proceso de desarrollo más equitativo y que tenga en cuenta la ampliación de sus capacidades.

En la perspectiva de desarrollo de Sen, la capacidad de la sociedad de poder generar formas de acceso y participación económica reales a toda la población, especialmente a los pobres, es imprescindible. Tal participación económica no implica solamente unos mayores ingresos para el consumo, sino la potencialización de sus capacidades y, en general, poder disponer mejor de su libertad según sus condiciones particulares. Es así que en una discusión con la teoría de Krugman, Amartya Sen interroga por la manera como 
se desvela la condición de desigualdad y segregación en una economía de aglomeración y cómo es posible la superación de tal condición.

Tal como se expondrá en el primer acápite, Krugman plantea divergencias de desarrollo entre lugares según las características económicas de estos. La desigualdad entre los espacios se explica por los procesos de aglomeración que conllevan el rezago de ciertas zonas por dar espacio histórico a la concentración de las fuerzas económicas en otras. La concentración de la industria posibilita desarrollo, y su lejanía a ella perpetúa la pobreza.

Ante la descripción que Krugman brinda de los procesos de desarrollo explicados por la concentración y la aglomeración, el autor no se compromete a plantear procesos de superación de tal disparidad. Desde su propio modelo no se evidencia interés de proponer una salida a la desigualdad. Parece que Krugman opta por adoptar la idea de "contagio" de las regiones pobres según se comuniquen a las regiones avanzadas. En su teoría no hay un tratamiento profundo del tema de la distribución de la riqueza socialmente producida, simplemente se deja como una posibilidad futura en el momento de convergencia entre las zonas atrasadas con las zonas de concentración económica. La desigualdad se encuentra entre lugares que generan procesos de aglomeración y economías a escala con las que no, o como en constantes momentos lo caracteriza Krugman: entre el mundo urbano y el mundo rural (cfr. Krugman 1991 y 1997)1.

Tal entendimiento del proceso de desarrollo en Krugman (y su eventual planteamiento de la desigualdad) está enmarcado en lo que Sen llama la visión más tradicional del pensamiento económico, que equipara desarrollo a crecimiento. Evidentemente, Krugman describe la importancia que trae para las economías el establecimiento de centros industriales ligados a rendimientos de escala que aceleran y aumentan el beneficio empresarial, y que conllevan un incremento en el agregado de la riqueza económica. Sin embargo, no es de su interés ahondar en el análisis justamente de las condiciones de vida que pueden llegar a tener los individuos durante dicho proceso. El desarrollo de una sociedad queda así reducido a la sombra del éxito industrial comprendido en su capacidad de generar economías de escala.

Desde una mirada diferente a la de Krugman, Sen sitúa el debate sobre la comprensión del desarrollo en las sociedades desde la perspectiva de los individuos que, dadas ciertas condiciones de su entorno y según sus características personales, pueden o no llevar una vida deseada. Es así que, si bien la riqueza social es, sin duda, un factor de desarrollo, no es el único elemento que permite hablar de un desarrollo de la sociedad. Al ubicarse

1 Si bien Krugman hace poca alusión a posibles superaciones de la disparidad económica entre diferentes lugares, el Informe del Banco Mundial de 2009 complementa las concepciones que desde la geografía económica se plantean sobre el tema de la desigualdad y el desarrollo. Tal presentación del Informe del Banco Mundial fue necesario incluirlo en el capítulo sobre Krugman para lograr mayor entendimiento del tema que nos concierne. 
desde el punto de vista de los individuos, se observan diferencias interpersonales que, aunque estando en conjunto en una situación agregada de la economía que puede ser favorable, no necesariamente permiten condiciones de vida adecuadas para todos. Para Sen, el desarrollo de la sociedad es una expresión del desarrollo de los individuos desde sus particularidades.

Bajo esta comprensión del desarrollo, para Sen es claro que la desigualdad económica corresponde a la forma como se ha desenvuelto la economía de mercado, mas no por una estructura determinista de la economía. Es por eso que es posible mejorar las dificultades de los más rezagados económicamente por medio de un enfoque diferente dentro de la misma economía de mercado sin necesidad de plantear un sistema económico alternativo. De esta manera, su propuesta distributiva como posibilidad de equidad no espera un tiempo de convergencia del devenir económico, sino acciones directas desde la política para impulsar la reducción de la disparidad.

En el marco del reconocimiento tanto de la validez como de las diferencias entre los enfoques de los dos autores es plausible reflexionar sobre la posibilidad de una propuesta argumentativa y metodológica que incorpore elementos propios y auténticos de las tesis de las dos teorías. En un contexto de aglomeración económica (Krugman), ¿se puede generar un proceso de desarrollo humano (Sen)? O, en otras palabras, ¿puede la propuesta de desarrollo humano de Sen desplegarse en las condiciones de desarrollo que describe Krugman?².

Las respuestas a estas preguntas conllevan el desarrollo de la presente tesis en dos secciones esenciales para la comprensión adecuada de las teorías de Krugman y Sen, respectivamente, en lo concerniente a sus concepciones de la noción de desarrollo, para así poder proponer, en un tercer acápite, la construcción de un modelo dinámico simple que incorpore los elementos descritos de las teorías. Tal modelo sencillo define una función del consumidor y recoge las ideas de Sen al hablar de "agentes" que toman decisiones, pero ubicados en una realidad de disparidad económica que hemos optado por llamar desigualdad en las circunstancias, que Krugman describe como el proceso de economías de aglomeración. Como el lector observará, fue necesario plantear un índice de segregación que expresa la condición de desigualdad entre dos ubicaciones diferentes (tomando los principios de Krugman) y a partir de ahí realizar impactos de política pública (basándonos en Sen) que conducen a una distribución fiscal, logrando así una mayor paridad de condiciones de vida entre las dos ubicaciones.

2 Como experiencia de investigación que logra relacionar principios teóricos de Krugman y Sen, se toma como referente el Informe de Desarrollo Humano para Bogotá. Bogotá, una apuesta por Colombia, (PNUD 2008), en el que se elabora una propuesta de "índice de desarrollo urbano" integrando elementos de la nueva geografía económica (como lo son la "densidad" y la "distancia") con el índice de desarrollo humano clásico basado en Sen. 
La coherencia del modelo permite situarse desde la perspectiva del consumidor, de las personas y sus condiciones, y no desde el enfoque de las empresas, tal como lo realiza Krugman. El proceso de aglomeración genera mejores condiciones de vida para quienes viven en las zonas industriales, desde el enfoque de las capacidades. La propuesta es, pues, cómo extender esas posibilidades para las zonas segregadas. Como resultado se puede observar una distribución económica que aumenta las capacidades de los hogares de la zona menos desarrollada dentro del modelo hipotético que se plantea. La profundización de estas consideraciones y conclusiones se plantean en la última sección.

\section{El proceso de aglomeración: Lectura de Paul Krugman}

"Por geografia económica entiendo la localización de la producción en el espacio, es decir, la rama de la economía que se preocupa de dónde ocurren las cosas”.

(Paul Krugman, Geografia y comercio, p.7)

Es el mismo Paul Krugman quien en su libro Geografia y comercio (1993) sitúa de manera comparativa la relación que puede establecerse entre la noción de desarrollo y el estudio de la economía en perspectiva espacial. La propuesta que realiza en tal libro es precisamente el resultado de reflexionar conjuntamente la preocupación central de las teorías del desarrollo - "explicar por qué algunos países son mucho más pobres que otros" (p. 8)-, con los adelantos y las limitaciones de la entonces geografía económica, centrada en entender el porqué de la "localización de la producción en el espacio" (p. 7)3. Justamente las investigaciones que se realizan en el marco de una teoría económica que tenga en cuenta la dimensión espacial puede (o debe) contribuir a entender esas diferencias entre las regiones desarrolladas y las que no, y de los procesos que generan y reproducen tal disparidad.

En esta perspectiva, la geografía económica del momento lograba hacer algunas aproximaciones sobre la explicación de las razones de las diferencias económicas (desarrollo) entre las regiones, basándose, entre otras cosas, en la teoría de ventajas comparativas, las condiciones de "primera naturaleza" del territorio (clima, proximidad a mares y ríos, etc.) o la misma historia, que conllevaba el asentamiento humano y sus diferentes actividades en ciertos lugares.

Sin embargo, pese al reconocimiento de ideas innovadoras de la geografía económica, Krugman (1997) identifica varias limitaciones en su incapacidad para modelar y sustentar matemáticamente sus explicaciones, al tiempo de tener vacíos explicativos en ciertos temas, por ejemplo, los procesos de aglomeración y la imposibilidad de poder asimilar

3 Tal forma de introducir su teoría a partir del acercamiento de las teorías del desarrollo con las teorías de geografía económica también se aprecia en el libro Desarrollo, geografia y teoría económica (1997), en el que hace una genealogía un poco más extensa de las dos corrientes al principio del texto. 
elementos importantes de la teoría económica moderna, como la competencia imperfecta o el mecanismo de fijación de precios.

Es así como la nueva geografía económica (NGE) aparece como teoría que incorpora elementos adicionales a la teoría tradicional de geografía económica y a la teoría clásica de localización, tales como la toma racional de decisiones que fortalecen la fundamentación microeconómica, la condición de rendimientos crecientes, la aplicabilidad de equilibrio general dentro del modelo y la profundización de la noción de "externalidades" como posibilidad de derivar de manera endógena los factores que generan o impiden los procesos de aglomeración.

Esta serie de elementos y estrategias que incorpora Krugman -entre otros pensadores, como Fujita y Venables- logra situar a la NGE como un corpus teórico que permite aumentar la comprensión de los procesos que se despliegan en el ordenamiento geográfico de la economía.

No obstante, se debe entrar un poco más en detalle para analizar los alcances que tal teoría plantea sobre desarrollo, en el sentido que el mismo Krugman ya ha descrito, a saber: desarrollo es la condición de no pobreza. ¿Cómo explica Krugman desde la NGE los procesos económicos que llevan a una región a ser pobre y a otra región a ser desarrollada? ${ }^{4}$

\subsection{Economías de aglomeración}

Tal como se comentó con anterioridad, los teóricos e investigadores del desarrollo parten de una pregunta originaria: ¿por qué unos países son más pobres que otros? Con el adicionamiento de la interpretación espacial, la pregunta adquiere un nuevo matiz: ¿por qué el desarrollo se concentra en ciertos lugares? ${ }^{5}$

Según Krugman, son varios los factores que se entrelazan dentro de las dinámicas económicas que permiten o no la ubicación de la producción en algún lugar determinado y, con ello, la posibilidad de desarrollo . Krugman agrupa tales factores de manera genérica como "fuerzas (o externalidades) centrípetas" y "fuerzas (o externalidades) centrífugas" , que posibilitan o limitan los procesos de aglomeración (ver tabla 1).

4 Como prolegómenos a lo que sería la obra maestra de la nueva geografía económica, presentada en el Informe sobre desarrollo humano 2009, el Banco Mundial empieza un artículo del año 2000, titulado "Geography and development" de la siguiente manera: "Why do these spatial inequalities exist, and why are spatial diference in land rents and wages (...) why is economic activity so concentrated?" (p. 1).

5 Si bien la investigación de Krugman lo lleva a identificar y explicar los factores que han permitido concentrar en un espacio determinado un conjunto de empresas, en varias ocasiones habla de "accidentes de la historia" que, por cuestiones azarosas o decisiones individuales sin mayor explicación, permitieron en varias ocasiones el inicio de la ubicación de las empresas en el lugar correspondiente (Ver Krugman, 1993, p. 110).

6 Ver Geografía y comercio, apéndice A, y primer capítulo (especialmente la p. 47) de Desarrollo, geografía y teoría económica. 
Tabla 1. Fuerzas que afectan la concentración y la dispersión geográfica

\begin{tabular}{l|l}
\multicolumn{1}{|c|}{ Fuerzas centrípetas } & \multicolumn{1}{c}{ Fuerzas centrífugas } \\
\hline Enlaces & Inmovilidad de factores \\
\hline $\begin{array}{l}\text { Densidad de los mercados } \\
\begin{array}{l}\text { Difusión del conocimiento y otras externalidades } \\
\text { económicas puras }\end{array}\end{array}$ & Renta tierra / desplazamientos \\
\hline
\end{tabular}

$$
\text { Fuente: Fujita y Krugman (2004). }
$$

Dentro de las fuerzas centrípetas se comprenden, en primer lugar, los "enlaces" que generan las empresas al establecerse en un lugar, correspondientes a las "conexiones" o a los "encadenamientos hacia atrás o hacia adelante" que Hirschman propone y que posibilitan el aumento de la demanda y la reducción de los costos de producción. Un encadenamiento hacia atrás eleva la demanda de otra actividad, por ejemplo, un aumento de la producción de acero puede elevar la demanda de carbón. Un encadenamiento hacia adelante reduce el costo de producción de otra actividad (un aumento de la producción de acero reduce el coste de la producción de barcos).

En segundo lugar, y retomando a Marshall en su concepto de economías externas, se identifica como fuerza centrípeta la búsqueda de las empresas de establecerse en lugares densos donde haya gran demanda del mercado y en donde se pueda encontrar disponibilidad especializada de trabajadores, ventajas que propicia la concentración de las empresas en un mismo sector.

Por último, se comprenden como otras fuerzas centrípetas las externalidades tecnológicas, tales como el flujo, la transferencia e imitación de información, que posibilitan el mejoramiento de productividad de las empresas.

Por el contrario, dentro de las fuerzas centrífugas se puede comprender la no movilidad de factores como la tierra (y su costo) o el trabajo (en donde hay impedimentos de migración), que tiende a llevar a las empresas a ubicarse en lugares donde tales factores sean más económicos, dispersando el devenir de la aglomeración. Junto con ellos existen externalidades negativas, como la congestión, la contaminación y la seguridad, que llevan a las personas y a las empresas a buscar lugares diferentes a los de aglomeración ${ }^{7}$.

La historia que se desenvuelve entre la relación de las fuerzas centrífugas y las centrípetas origina los niveles y las formas de aglomeración entre lugares. El equilibrio económico generado entre estos dos tipos de fuerzas es desarrollado por Krugman originariamente en un modelo básico de dos zonas, en el que la estructura del mercado queda establecida

7 Como se observará más adelante, estas externalidades negativas son de espacial interés y cuidado al momento de reflexionar sobre los alcances y las limitaciones del proceso de desarrollo en las economías de aglomeración. 
en una relación de centro-periferia, donde el centro es el lugar en el cual se despliega la aglomeración y la periferia no ${ }^{8}$. El "centro" es el lugar donde se concentra la producción económica y sus correspondientes efectos, tales como la urbanización, la tecnificación, la especialización, etc. Por lo demás, la periferia termina siendo rezagada en función del centro. Krugman comprende esta relación centro-periferia como la relación entre el mundo urbano y el rural, entre aquellas regiones, países, ciudades que han logrado cierto nivel de industrialización y producción y aquellos que no; en definitiva, entre los lugares desarrollados y los que no, es decir, los pobres.

\subsection{Desarrollo en economías de aglomeración}

La visión de la nueva geografía económica parte de la premisa de que los centros industriales y empresariales son los núcleos del crecimiento económico en tanto se convierten en los espacios, por antonomasia, de las sociedades modernas donde se concentra la producción, y con ello se configura el mercado tanto espacial como económicamente. Sin embargo, dicha concentración, tal crecimiento económico, ¿qué tipo de desarrollo desencadena para el conjunto de la sociedad? ¿Cómo repercute en la vida de los individuos?

Según el Informe sobre Desarrollo Mundial de 2009, la aglomeración ocasiona un efecto sumamente importante en la economía, a saber, la capacidad de establecer, acceder e integrar mejor al mercado.

Los núcleos industriales (“centros de producción”) se establecen por la intención de reducir los costos al tiempo de aumentar beneficios: buscan lugares de alta demanda, donde puedan disminuir costos por uso del suelo y de transporte. Cada empresa calcula la oportunidad de establecerse en otro lugar dependiendo inversamente de los costos de transporte. Es así que el núcleo de producción termina generando una red de intercomunicación con la vecindad según las entradas por concepto de materias primas y bienes intermedios para la producción, o según el orden de distribución de su producto. De esta manera, tanto el centro como la periferia gozan de dicho sistema de mercado.

De igual manera, el mercado de trabajo tiende a especializarse y a tener movilidad según se van estructurando los nodos de producción. Los centros industriales llaman tanto a la concentración empresarial como a la demográfica, originando así la aglomeración. El flujo de fuerzas que conducen a tal aglomeración plantea un argumento circular (“causación circular y acumulativa"): las empresas se ubican en mercados de demanda

8 Se han desarrollado otras ampliaciones al modelo centro-periferia de Krugman, en el que se intenta llevar la reflexión a un mayor número de dimensiones. Por ejemplo, se conoce la introducción al Modelo de Enfoques Evolucionistas, en el cual los espacios de localización se entienden como una línea recta distribuida uniformemente en la tierra y en donde los agentes toman las decisiones - CFC Fujitay Krugman (2004) -. El presente trabajo desarrolla las ideas centrales del Modelo original de Doble-Localización (centro-periferia), que será abordado en mayor detalle en la tercera sección. 
grande (abundancia de consumo y mano de obra), pero, así mismo, los hogares tienden a buscar los lugares donde las empresas están ubicadas (posibilidad de trabajo) y donde haya variedad de producción.

La aglomeración por efecto económico impulsa todo un desarrollo administrativo, institucional, político y cultural que construye sociedad. Tanto las empresas como los trabajadores requieren de un complejo de incentivos que brinden seguridades a las primeras y un conjunto de requerimientos que posibilitan la integración y bienestar social para los segundos 9 .

El proceso de aglomeración genera varias externalidades positivas para la población donde la producción se concentra. No solo las empresas se ven beneficiadas, sino también la sociedad en su conjunto. Por ejemplo, la concentración y el crecimiento de la población implica consolidar instituciones que garanticen seguridad de la propiedad y protección social. Los centros económicos generan una variedad de bienes públicos y de equipamientos colectivos que propician desarrollo de las comunidades.

Pero así mismo, las ventajas empresariales generan ciertas externalidades negativas tanto económicas como sociales. Por un lado, es notable la disparidad que se genera entre las zonas industriales y las que terminan por ser rezagadas a la periferia. La pobreza se localiza proporcionalmente a la oportunidad de cercanía del centro. Y, por otro lado, dentro del mismo proceso de concentración se cae en el riesgo de generar viciosas dinámicas de aglomeración que tienen que ver con la excesiva densidad de la población (propia de las migraciones hacia el centro), la congestión, la contaminación y la criminalidad (PNUD, 2008).

Retomando la concepción de desarrollo como superación de la pobreza -el rezago dentro de las sociedades-, surge la pregunta hacia el proceso que Krugman ha descrito entre la separación de lugares industrializados y rezagados: ¿permite la aglomeración generada por la concentración de la producción generar un tipo de desarrollo que supere la pobreza?

9 El Informe del Banco Mundial (2009) agrupa en tres categorías tales elementos de política que deben acompañar el proceso de aglomeración, a saber: instituciones, infraestructuras e intervenciones. "Instituciones: término sinóptico utilizado en este Informe para hacer referencia a políticas que son espacialmente neutras en su diseño y deberían ser de cobertura universal. Algunos de los principales ejemplos son los reglamentos que repercuten en la tierra, la mano de obra y el comercio internacional, y servicios sociales como la educación, la salud y el abastecimiento de agua y el saneamiento, financiados con impuestos y mecanismos de transferencia. Infraestructura: término sinóptico utilizado aquí para hacer referencia a políticas e inversiones espacialmente integradoras. Como ejemplos cabría citar las carreteras, los ferrocarriles, los aeropuertos, los puertos y los sistemas de comunicaciones que facilitan la circulación de bienes, servicios, personas e ideas en el plano local, nacional e internacional. Intervenciones: término sinóptico aquí utilizado para hacer referencia a los programas espacialmente focalizados que muchas veces dominan el debate sobre políticas. Como ejemplos cabría citar los programas de eliminación de barrios de tugurios, los incentivos fiscales para las empresas manufactureras ofrecidos por gobiernos estatales y el acceso preferencial al comercio para los países pobres en los mercados de los países desarrollados." 


\subsection{Desigualdad en la aglomeración}

Tal como se ha venido comentando, el proceso de aglomeración genera en sí mismo una desigualdad económica ubicada espacialmente, dependiendo del acceso o de la cercanía al lugar donde la industrialización se esté generando. La pobreza no es solo la ausencia de crecimiento industrial o de economía a gran escala, sino también de imposibilidad de acceso a los lugares de crecimiento. La pobreza se perpetúa por la desigualdad entre los espacios con crecimientos dispares y por su falta de vinculación. De ahí que el Informe del Banco Mundial, siguiendo a teóricos de la nueva geografía económica, aborda el problema de la superación de la desigualdad, y con ello de la pobreza, a partir de la noción de "contagio" o "integración", términos empleados, a su vez, por Paul Krugman.

El crecimiento económico generado por un proceso de aglomeración evidencia consecuencias de desigualdad geográfica según se ubiquen los centros de producción y de periferia. La desigualdad se manifiesta entre el mundo urbano y el mundo rural dentro de los países; y en las ciudades, la pobreza queda desplazada a los anillos más externos de estas.

Según el informe, esta realidad debe ser manejada con mucho cuidado por los agentes de política. No se trata de impedir el crecimiento desigual, sino de posibilitar el contagio y la conexión de las zonas atrasadas a los centros de producción.

La prosperidad no llega a todos los lugares a la vez, pero ningún lugar debe quedar condenado a la pobreza. Con políticas acertadas, la concentración de la actividad económica y la convergencia de los niveles de vida pueden producirse a la vez. El desafío que se presenta a los gobiernos es permitir - e incluso alentar- un crecimiento económico "desequilibrado" y, al mismo tiempo, garantizar un desarrollo incluyente. (Banco Mundial, 2009, p. 20).

De esta manera, el informe hace clara la diferencia entre tres tipos de desigualdades, a saber: 1) desigualdades espaciales por actividad y producción económica, 2) desigualdades espaciales en niveles de vida y 3) desigualdades sociales (cfc. Recuadro 0.3 del Informe). Las primeras obedecen al propio proceso de aglomeración centralizada en ciertos lugares, tal como se ha comentado. Tales desigualdades las establecen las fuerzas del mercado y no deben ser interrumpidas. Por su parte, las desigualdades sociales y por niveles de vida, si bien son costos del proceso inicial de aglomeración y de crecimiento centralizado, a largo plazo llegarán a un equilibrio conforme la integración se genere adecuadamente.

El éxito de la integración dependerá de las estrategias y del buen desempeño de los gobiernos al momento de colaborar - mediante buenas políticas de establecimiento y promoción de "instituciones", "infraestructuras" e "intervenciones"- al proceso libre de concentración de la producción y aglomeración de las sociedades. "La mejor manera de conseguir esta integración es dar rienda suelta a las fuerzas del mercado de la 
aglomeración, la migración y la especialización, en vez de combatirlas u oponerse a ellas" (Banco Mundial, 2009, p. 20).

La situación de competencia imperfecta y los rendimientos crecientes ponen en duda la posibilidad de "convergencia" en el sentido tradicional de la teoría neoclásica. El proceso de aglomeración no se da manera uniforme sobre el territorio debido a la centralización de la producción y a la "causación acumulativa y circular" que se genera. De ahí que el desarrollo desde la perspectiva de la NGE se establezca en términos de "contagio" o de integración. La finalidad de la integración o el contagio no es distribuir la producción ni descentralizar la economía. El objetivo es ampliar a las zonas periféricas los niveles de vida logrados por efecto de la aglomeración. Tal nivel de vida se puede conseguir con políticas focalizadas dirigidas a fortalecer las instituciones, ampliar las infraestructuras y hacer intervenciones orientadas a grupos poblacionales vulnerables.

\section{Propuesta de desarrollo humano de Amartya Sen}

"Los trascendentales poderes del mecanismo de mercado han de complementarse con la creación de oportunidades sociales básicas para conseguir la equidad y justicia social".

(Amartya Sen, Desarrollo y libertad, p. 179)

\subsection{Desarrollo como libertad}

Desde otra perspectiva sobre el entendimiento del desarrollo, Amartya Sen concibe una teoría centrada en las condiciones de vida de los individuos. Con profundo interés en los temas de elección social - entendida como la forma en la que los individuos de la sociedad toman decisiones colectivas teniendo en cuenta sus valoraciones y condiciones individuales-, Sen sitúa el problema del desarrollo como la posibilidad social de mejorar las condiciones de vida de los individuos.

La teoría de Sen es profundamente crítica de las nociones de desarrollo que lo comprenden únicamente como crecimiento económico. Para Sen, el desarrollo va más allá de fines rentistas o de acumulación de riqueza, etc. El desarrollo abarca un conjunto de posibilidades de realización para las personas, que indiscutiblemente tienen que ver con su capacidad adquisitiva, pero que la superan.

Tan importante es reconocer el papel fundamental que desempeña la riqueza en la determinación de las condiciones de vida y de la calidad de vida, como comprender el carácter limitado y eventual de esta relación. Una concepción satisfactoria del desarrollo debe ir mucho más allá de la acumulación de la riqueza y del crecimiento del producto nacional bruto y de otras variables relacionadas con la renta. Sin olvidar la importancia del crecimiento económico, debemos ir mucho más allá. (Sen, 2000, p. 30). 
La riqueza o la renta en sí mismas no pueden ser el criterio, el índice que mida cabalmente el nivel de desarrollo de las sociedades, según Sen, porque hay una gran variedad de factores y condiciones que relativizan el valor de la riqueza entre individuos y sectores poblacionales dentro de la misma sociedad. Un mismo salario, por ejemplo, será asumido y utilizado de diferente manera por un enfermo y por una persona sana, puesto que las posibilidades de uso de los ingresos estarán determinadas según sus condiciones de vida.

Tal agregado de posibilidades de elección, que en términos generales Sen llama "libertad", se amplía (siendo ello el objetivo) según el conjunto de "capacidades" reales que tienen los individuos al momento de tomar sus elecciones persiguiendo sus objetivos de realización personal. Así se define el concepto de desarrollo humano estructurado por Sen según el Informe de Desarrollo Humano de 2008:

Se entiende por desarrollo humano la ampliación de las capacidades de las personas (...) El conjunto de capacidades de una persona se puede definir como el conjunto de vectores de realizaciones a su alcance. Si el número de vectores aumenta, es decir, si el conjunto de realizaciones es mayor, las capacidades se amplían. (PNUD, 2008, p. 308).

Con el aumento de capacidades del individuo se alcanza mayor grado de libertad y, con ello, mayor nivel de desarrollo. En definitiva, el éxito del desarrollo se determina por la posibilidad de las sociedades de hacer a sus hombres y mujeres personas libres. El desarrollo se basa en la libertad justamente porque esta permite a los individuos aumentar las capacidades que les permitan vivir de la forma en que quieran vivir, lo cual es, según Sen, el objetivo de alcanzar un mayor desarrollo. En palabras del nobel: "el desarrollo puede concebirse (...) como un proceso de expansión de las libertades reales de las que disfrutan los individuos" (Sen, 2000, p. 19).

\subsection{Pobreza y desigualdad}

En sus diferentes textos, Amartya Sen entra en debate con la concepción tradicional y popularizada de que la pobreza es un asunto de posibilidad adquisitiva de bienes, de cierto nivel adecuado de renta o ingresos. Apelando a la tradición de pensamiento occidental, desde Aristóteles hasta Adam Smith, Sen cuestiona la simpleza de dicha concepción. La riqueza no se quiere por sí misma, no es un fin como tal, sino un medio, un instrumento para alcanzar otros bienes. En cuanto medio, está condicionado por una serie de factores adicionales que posibilitan a una persona no solamente ser no-pobre, sino también alcanzar los objetivos de su proyecto de vida.

Entre tales factores se encuentran, por ejemplo ${ }^{10}$ :

1. Heterogeneidad de condiciones personales

10 Cfc. Sen, Desarrollo y libertad, p. 94. 
ISSN: 0124-3551 / Año 16, No 25 / julio-diciembre / pp. 43-70

2. Diversidad relacionada con el medio ambiente

3. Diferencias del clima social

4. Diferencias entre las perspectivas relacionales

5. Distribución dentro de la familia.

Una simple comparación interpersonal evidencia que dos personas con una renta igual pueden tener una calidad de vida de diferente, esto según sus circunstancias personales, su entorno ambiental, político, económico y hasta familiar. Por esta razón, Amartya Sen no puede sostener su enfoque de desarrollo como superación de la pobreza solamente a partir de la renta o, en términos absolutos, de la riqueza.

Para Sen, "la pobreza debe concebirse como la privación de las capacidades básicas y no meramente como la falta de ingresos, que es el criterio habitual con el que se identifica la pobreza" (Sen, 2000, p.114). Evidentemente, eso no rechaza la idea de la importancia de la renta, pues la carencia de un buen nivel de ella imposibilita, en muchos casos, el logro de las capacidades.

$\mathrm{Al}$ igual de lo que Sen denomina "pobreza real", que complementa la noción de pobreza de renta, el autor dirige la discusión a la noción, también limitada, de desigualdad. Desde el punto de vista de las libertades como capacidades efectivas de cumplir el propósito de vida, Sen hace de nuevo la distinción entre "desigualdad de renta" y "desigualdad económica", según la cual esta última involucra otras realidades complementarias de la renta que son factores de privaciones de capacidades, como lo son "el paro, la falta de salud, la falta de educación y la exclusión social” (Sen, 2000, p.138).

(...) la distinción entre la desigualdad de la renta y la desigualdad económica es importante. (...) Por ejemplo, es posible pensar que dar una proporción mayor de la renta a una persona que tiene más necesidades -debido, por ejemplo, a una incapacidad-va en contra del principio de la igualdad de las rentas, pero no va en contra de los preceptos más amplios de la igualdad económica, ya que cuando se valoran los requisitos de la igualdad económica, hay que tener en cuenta que esta persona necesita más recursos económicos a causa de su incapacidad. (Sen, 2000, p.138).

Ampliando los conceptos de pobreza y desigualdad, Amartya Sen entra en debate con las nociones tradicionales que privilegian la noción de renta como el criterio único y fundamental para medir los niveles económicos individuales y sociales. Como se ha mencionado varias veces, Sen es un gran crítico de las posturas que defienden el mecanismo de mercado en sí mismo y que obvian los efectos negativos que puede traer.

Como veremos, sus nociones de "pobreza real" y "desigualdad económica", basados en la noción de capacidades efectivas de realización de los individuos, le motivan a plantear, en consecuencia, una propuesta de "complementar" el mercado para lograr el desarrollo como libertad de individuos y países. 


\section{3 "Bienes públicos" como factores de desarrollo}

Como hemos visto, Amartya Sen amplía la concepción tradicional de la pobreza basada en carencia de renta y expone la "necesidad de adoptar un enfoque pluralista" para la comprensión de la desigualdad.

Desde el enfoque de carencias de capacidades para comprender la pobreza y la desigualdad, la concepción de desarrollo que conlleva justamente la superación de las privaciones adquiere nuevos matices. El desarrollo de una sociedad podrá medirse según el grado que pueda aumentar las capacidades de los individuos. Si bien ello implica cierto nivel de renta, también depende de una serie de factores adicionales.

Así, por ejemplo, Sen (2000) considera que hay factores de desarrollo que están (o deben estar) por encima de la lógica del mercado, ya sea por sus características de consumo conjunto por la sociedad o porque representan un beneficio para todos. Tales tipos de factores de desarrollo se llaman "bienes públicos".

Todo puede comercializarse (si queremos llevarlo al mercado), y no hay nada que influya de manera significativa en nuestro bienestar que no pase por el mercado. Sin embargo, en realidad algunos de los bienes que más contribuyen a las capacidades humanas pueden ser difíciles de vender exclusivamente a una persona cada vez. Es el caso, sobre todo, de los llamados bienes públicos, que no son consumidos por separado, sino conjuntamente por todos los individuos. (Sen, 2000, p.161).

Dentro de los bienes públicos ${ }^{11}$, Sen agrupa, por ejemplo, factores de asistencia sanitaria, conservación del medio ambiente, salubridad pública y cuidado epidemiológico. "Una persona puede estar dispuesta a pagar lo que le corresponde de un programa social de erradicación de la malaria, pero no puede comprar su parte de esa protección como si fuera un "bien privado" (por ejemplo, una manzana o una camisa). Es un bien público - un entorno libre de malaria- que tenemos que consumir conjuntamente" (Sen, 2000, p. 162).

De igual manera, para Sen hay otra serie de bienes que son de beneficio privado, pero cuya disponibilidad general ayuda al beneficio colectivo. Tal es el caso, por ejemplo, de la educación básica. Es un bien que no solo amplía las capacidades individuales, sino también las colectivas para el progreso social.

(...) la educación básica también puede tener un componente de bien público (y puede concebirse como un bien semipúblico). Las personas que reciben educación se benefician, por supuesto, de ella, pero, además, una expansión general de la educación y de la alfabetización en una región puede facilitar el cambio social (...) y contribuir también a mejorar el progreso económico, del cual otros también se benefician. (Sen, 2000, p.162).

11 Tal como se observa, la concepción de Sen de "bien público" contempla sus características de no rivalidad y no exclusión. La primera cualidad es entendida como el uso del bien por un individuo sin que ello afecte el uso simultáneo por otro individuo; y la segunda cualidad, como el no impedimento de su uso y satisfacción por cualquier individuo. 
La existencia de tales tipos de bienes conduce a Sen a defender la idea de la intervención del Estado para impulsar dichos factores de desarrollo en el proceso continuo e ideal de aumentar las capacidades de la sociedad. La posibilidad de ampliación de tales bienes públicos en las zonas marginadas permite el desarrollo de una manera más equitativa y ayuda a incrementar las condiciones reales de los individuos en el mejoramiento de su calidad de vida. La intervención del Estado, en este orden de ideas, será efectiva para disminuir la desigualdad entre las capacidades de los individuos al tiempo de no obstaculizar el desarrollo de la sociedad en su conjunto.

El desarrollo humano es un aliado de los pobres más que de los ricos y los opulentos. ¿Qué hace el desarrollo humano? La creación de oportunidades sociales contribuye a la expansión de las capacidades humanas y a la mejora de la calidad de vida. (...)

Como hemos visto, el desarrollo humano no solo mejora la capacidad de vida, sino también influye en las capacidades productivas de los individuos y, por tanto, en el crecimiento económico, beneficiando a todo el mundo. (Sen, 2000, pp. 180-181).

\section{Aumento de capacidades en economías de aglomeración}

\subsection{Recopilación conceptual de las teorías}

Con enfoques conceptuales heterogéneos, Krugman y Sen han abordado el problema del desarrollo - cómo un lugar supera la condición de pobreza- con perspectivas reflexivas diferentes. Krugman explica y describe el proceso de crecimiento económico generado por la "causalidad circular y acumulativa" de la aglomeración que concentra la actividad económica en unos lugares, haciendo posible el crecimiento productivo con economías de escala. Sin embargo, el proceso de concentración guarda consigo una condición de aislamiento de los centros económicos que conlleva a que lugares periféricos y distantes no gocen los beneficios y desarrollos de la aglomeración. Tal como lo describe Krugman, el desarrollo generado por el proceso de aglomeración produce desigualdades económico-espaciales en las cuales (ver Informe del Banco Mundial de 2009) las condiciones de vida de las personas son diferentes. La nueva geografía económica de Krugman explica, tal como el autor se lo propuso, por qué unos países (regiones, territorios, ciudades, etc.) son ricos y otros quedan inmersos en la pobreza.

La potencia explicativa de la teoría de Krugman (y su desarrollo en otros autores), que ha diseñado modelos de equilibrio mediante la relación de lugares con condiciones económicas desiguales, cuestiona (e invita al pesimismo) sobre la posibilidad de convergencia de los lugares pobres y ricos. La posibilidad de desarrollo cualitativo de los lugares pobres solo es posible por la capacidad de conexión que tengan con los centros productivos. 
Ante esta realidad concluyente de la teoría de Krugman (donde, por ejemplo, en el Informe del Banco Mundial se afirma taxativamente que los gobiernos deben acompañar los procesos de concentración económica haciendo políticas focalizadas de disminución de pobreza sin que se interrumpan las fuerzas de aglomeración) se propone la teoría de Amartya Sen como un complemento necesario que invita a una reflexión comprometida a intentar superar, con acciones afirmativas de política, las condiciones de pobreza y desigualdad producidas por las dinámicas del mercado

Tal como se observó en la exposición de la segunda sección de la presente tesis, Sen es crítico con las concepciones que dejan a las fuerzas del mercado ser las ordenadoras, sin más, del desarrollo de las sociedades. Su teoría, al reflexionar sobre las condiciones de vida de los individuos, plantea recursos adicionales que deben acompañar el crecimiento económico que generan las decisiones empresariales en un contexto de libre mercado, tales como el fortalecimiento y la inversión del Estado en bienes públicos.

La introducción de Sen y su concepto principal de desarrollo como ampliación de capacidades de los individuos en un contexto de aglomeración es pertinente en tanto es el mismo Sen quien afirma que su propuesta no sale de la realidad del libre mercado, sino que es justamente en un contexto de libertades donde se puede comprender y materializar el desarrollo como libertad. El valor de la teoría de Sen es precisamente que el desarrollo es teorizado desde la perspectiva de los individuos y no desde el crecimiento económico de las empresas.

Es así que en un contexto de relaciones económicas entre fuerzas centrífugas y centrípetas que posibilitan aglomeración, las acciones de política que generan desarrollo humano deben permitir aumentar las condiciones de vida de aquellos que han quedado marginados o segregados económica y espacialmente por las dinámicas del mercado.

Como es de esperarse, el éxito de relacionar a Sen con Krugman debe tener supuestos básicos en los que se puedan recoger mínimamente las dos teorías, evitando así forzar los planteamientos de los autores. A continuación, se presenta tal propuesta, que consta, de manera general, de dos momentos. En primer lugar, se construye formalmente una función del consumidor que busca maximizar su utilidad teniendo en cuenta tanto sus condiciones particulares como las características del entorno. Tales elementos, como se observará, recogen las ideas centrales que se han expuesto de los dos autores.

Y en un segundo momento, de tal modelo formal construido se expone una simulación de comportamiento de los hogares utilizando la plataforma Netlogo, que es particularmente útil para modelar sistemas que evolucionan en el tiempo. Tal plataforma de simulación hipotética permite dar instrucciones a un número indefinido de agentes para que todos ellos operen de manera independiente, entre sí y con el entorno. Esto hace posible explorar la relación entre el comportamiento de los individuos y los patrones que 
surgen a partir de la interacción de muchos individuos entre sí. Al utilizar la plataforma Netlogo podemos justamente relacionar las capacidades de los hogares, en el sentido de Sen, con características del entorno en condición de aglomeración.

Sin pretender ser un modelo acabado, la propuesta dada a continuación permite proyectar analítica y formalmente la discusión teórica de los dos autores, y es también una invitación para el análisis y la discusión.

\subsection{Generalidades del modelo}

El modelo que se propone a continuación toma ideas básicas de aglomeración que Krugman desarrolla en los textos Geografia y comercio (1993) y Desarrollo, geografia y teoría económica (1997), en los que hace una comparación y establece la relación de un mundo cerrado con dos zonas de condiciones diferentes: una donde se concentra el proceso de aglomeración y otra zona que queda rezagada (Modelo Centro-Periferia). En esta realidad se analizan las conductas de los consumidores que buscan optimizar su utilidad según el concepto de capacidades de Sen. Afirmamos que la disparidad entre las dos zonas origina desigualdad y segregación, siendo necesario intervenir mediante acciones de política económica que propician paridad de niveles de vida. Con esta propuesta se estructura un esquema en la que el modelo de aglomeración se centre en las decisiones de los agentes que quieren mejorar sus niveles de vida (ampliar sus capacidades) y no desde las decisiones de las empresas al momento de optimizar sus beneficios. Tal modelo podría ampliar las visiones de la geografía económica desde una perspectiva de desarrollo humano.

El desarrollo del modelo intenta evidenciar las decisiones que toman los hogares cuando tienen características desiguales entre sí dadas sus condiciones particulares al momento de optimizar su utilidad (mejorar su calidad de vida). Tales decisiones están condicionadas, a su vez, por las características desiguales de dos zonas que se diferencian por el grado de desarrollo que tiene cada una según el proceso de aglomeración y que, por ende, también adquieren características diferentes. Las diversas capacidades propias de los hogares originan que algunos de ellos (los mejor dotados) se ubiquen en la zona desarrollada que, a su vez, por sus condiciones de desarrollo optimizan la utilidad del hogar. Por el contrario, las limitadas capacidades de los otros hogares aparecen como restricciones que los fuerza a situarse en la zona menos desarrollada, generando una función de utilidad inferior. De esta manera, dotaciones de los hogares y características de la zona guardan una relación circular propia de las economías de aglomeración. El desarrollo, pues, entendido en este modelo, incorpora las características de los lugares según un proceso de distribución desigual de características económicas (en el sentido de Krugman), junto con la posibilidad de aumento de las capacidades de los hogares, en el sentido de desarrollo humano de Sen. 
Las diferencias entre las dos zonas pueden entenderse por el conjunto de externalidades positivas y negativas del proceso de aglomeración, donde una de ellas (la desarrollada) ha acumulado una serie de bienes públicos que mejoran la calidad de vida de los hogares y ha consolidado un entorno favorable de vivienda y vecindad. Tal como se expuso en la primera parte, la aglomeración propicia el desarrollo de equipamientos y de seguridad social de las que las zonas rezagadas pueden carecer. Evidentemente, estas condiciones del entorno y su capacidad de disfrute tienen un costo para los hogares representado en el precio de la vivienda y de impuestos.

\subsection{Ecuaciones del modelo}

Partimos de una función de utilidad que cada hogar busca maximizar según su posibilidad de decisión en el lugar donde se encuentre $(\mathrm{h}=1,2)$.

$$
\text { Máx. } \mathrm{V}_{\mathrm{h}}^{\mathrm{i}}=\alpha_{\mathrm{x}}^{\mathrm{i}} \mathrm{x}_{\mathrm{h}}^{\mathrm{i}}+\beta_{\mathrm{h}}-\gamma_{\mathrm{p}}^{\mathrm{i}} \mathrm{p}^{\mathrm{h}}-\Phi\left(\mathrm{w}_{\mathrm{h}}^{\mathrm{i}}\right)(1)
$$

En la ecuación, $\alpha_{\mathrm{x}}^{\mathrm{i}}$ es un vector columna ( $\left.\mathrm{n} \times 1\right)$ que recoge las características del hogar, a saber: educación, salud y salarios, que funcionan como proxies de los elementos constitutivos del índice de desarrollo humano. Las características se distribuyen en el intervalo $[0 ; 1]$ de manera aleatoria, pero uniformemente, y para mayor significancia, en la generación aleatoria se pide una alta correlación entre ambos valores. De manera que, si la primera característica seleccionada fue salud, una persona con valores altos en este campo tiene mayor probabilidad de tener puntajes por encima de la media en salud.

El término $\mathrm{x}_{\mathrm{h}}^{\mathrm{i}}$ es un vector que le asigna a cada una de las características del hogar un coeficiente relacionado con las características de la zona donde se ubica la vivienda de cada hogar, $\mathrm{h}=1,2$. Los coeficientes de $\mathrm{x}_{\mathrm{h}}^{\mathrm{i}}$ varían en cada ronda de acuerdo con el conjunto de hogares ubicados en cada zona; es decir, entre más hogares den valor a la educación, el "vecindario" se ajusta y ofrece mejores características en esos aspectos. Como las características de las zonas son diferentes cualitativamente, se supone que a un hogar que se sitúe en la zona desarrollada se le asignarán coeficientes $\mathrm{x}_{\mathrm{h}}^{\mathrm{i}}$ más altos que en la zona segregada.

$\Phi\left(\mathrm{w}_{\mathrm{h}}^{\mathrm{i}}\right)$ es la función de recaudo de impuestos sobre el salario (que forma parte del vector de capacidades) que cumple $\Phi^{\prime}\left(\mathrm{w}_{\mathrm{h}}^{\mathrm{i}}\right)>0$ y $\Phi^{\prime \prime}\left(\mathrm{w}_{\mathrm{h}}^{\mathrm{i}}\right)<0$

El parámetro $\beta_{\mathrm{h}}$ es el acceso a los bienes públicos de cada región financiados por impuestos al salario de la región a la que pertenece. Entre menos disponibilidad tengan los hogares a los bienes públicos de la ciudad, menor será su utilidad. Por bienes públicos se entiende el conjunto de equipamientos, lugares e instituciones que son de acceso igual para todos los hogares de una zona y que posibilitan el aumento de capacidades de estas en el sentido de Sen. 
Por definición de bienes públicos tenemos que la no rivalidad y no exclusión pueden caracterizarse como:

$$
\beta_{\mathrm{h}}(\Phi)=\beta_{\mathrm{h}}{ }^{0}+\sqrt{\sum_{i=1}^{M} \phi\left(w_{h}{ }^{i}\right)}
$$

Como condición necesaria, la dotación de bienes públicos en las dos zonas inicia como un dato fijo, puesto que sin ese ajuste el proceso de elección de cada hogar (que depende de la decisión de todos los demás hogares) sería un proceso iterativo indeterminado.

Las dos zonas de la ciudad tienen precios de vivienda diferentes, $\mathrm{p}_{1}>\mathrm{p}_{2} \mathrm{P}_{1}>\mathrm{P}_{2}$, y el precio se ajusta en cada ronda según el promedio de los hogares de la zona. Por lo tanto, puede describirse una función de oferta de vivienda que determina los precios en cada región y el nivel de precios existente en cada ronda. Dado que la oferta es rígida, un aumento de la demanda de viviendas en la zona $\mathrm{h}$ en el período - $\mathrm{t}$ - se refleja únicamente en los precios en $t+1$, y su efecto permanece hasta que sea revertido por un descenso futuro de la demanda. De esta manera, $\gamma_{p}^{i}$ puede describirse como un ponderador del ajuste de los precios.

Con el modelo establecido, se tiene que un hogar (i) escoge localizarse en una de las dos regiones de la ciudad bajo las siguientes condiciones:

$$
\mathrm{E}\left(\mathrm{V}_{\mathrm{h} 1}^{\mathrm{i}}\right)>\mathrm{E}\left(\mathrm{V}_{\mathrm{h} 2}^{\mathrm{i}}\right)(3)
$$

Es decir:

$$
\alpha_{\mathrm{x}}^{\mathrm{i}} \mathrm{x}_{1}^{\mathrm{i}}+\beta_{1}-\gamma_{\mathrm{p}}^{\mathrm{i}} \mathrm{p}_{1}-\Phi\left(\mathrm{w}_{1}^{\mathrm{i}}\right)>\alpha_{\mathrm{x}}^{\mathrm{i}} \mathrm{x}_{2}^{\mathrm{i}}+\beta_{2}-\gamma_{\mathrm{p}}^{\mathrm{i}} \mathrm{p}_{2}-\Phi\left(\mathrm{w}_{2}^{\mathrm{i}}\right)(4)
$$

Es un modelo dinámico simple, en el que la dinámica viene dada por las iteraciones o rondas en el tiempo. De esta manera, se puede asociar el valor esperado del recaudo impositivo y, por lo tanto, la magnitud del bien púbico como la media aritmética del periodo inmediatamente anterior. Es decir:

$$
\mathrm{E}\left(\beta_{\mathrm{h}}(\Phi)\right)_{\mathrm{t}}=\beta_{\mathrm{h}}(\Phi)_{\mathrm{t}-1}(5)
$$

En la simulación, cada uno de los $(\mathbf{M})$ hogares se asocia con una de las dos zonas de la ciudad, creando así las condiciones para la siguiente ronda, que tendrá los mismos vectores, pero con los parámetros actualizados.

En el estado 0, las cualidades son asignadas aleatoriamente entre los agentes, con lo que se genera un primer estado de salarios, y luego los mismos elementos se van determinando bajo un proceso tal que su localización afecta sus capacidades (educación y salud) para generar ingresos (salarios). Es decir, la localización del hogar puede afectar positiva o negativamente las capacidades (salario, educación, salud), pero, al mismo tiempo, el salario es un factor que determina la elección de vivienda de los agentes. De modo que el modelo es dinámico, pero no muy complicado, y es coherente con las tesis de Sen. 
La intuición básica es que si el hogar tiene buenas capacidades y queda en una zona desarrollada (o industrializada en términos de Krugman), la ciudad "potenciará" las capacidades por medio del entorno y los bienes públicos, etc. De manera similar, si las capacidades del hogar son bajas y se establece en una zona desarrollada, puede ser que tarde o temprano sea relegado a la zona rezagada de la ciudad. De manera simétrica ocurre en el caso de iniciar en una zona rezagada de la ciudad. Esto genera una dinámica de segregación o convergencia que será más o menos acelerada debido a los parámetros del modelo.

Una vez establecida la determinación del salario (una función en cada ronda de las capacidades y de la localización), se procede a ordenar a cada agente de acuerdo con su nivel de salarios:

$$
w_{1}^{h}<w_{2}^{h}<\ldots<w_{h}^{M}
$$

Luego se calcula un salario promedio:

$$
w_{h}^{x}=\frac{\sum_{i=1}^{M} w_{h}^{i}}{M}
$$

Una vez establecido el salario promedio por ronda, se finaliza al determinar el índice de segregación calculando los salarios por ronda y graficando los datos correspondientes.

El índice de segregación es:

$$
\Phi=\frac{1}{2}\left[\sum_{h=1,2}\left(\frac{I_{h}^{-}}{I^{-}}-\frac{I_{h}^{+}}{I^{+}}\right)\right]
$$

El índice de segregación es un indicador que mide la diferencia entre la distribución de los dos grupos conformados entre los que tienen salario por encima y por debajo del promedio, donde $\mathrm{I}_{\mathrm{h}}^{-}$son las personas en la región h que tienen un salario inferior al promedio. $\mathrm{I}_{\mathrm{h}}^{+}$son aquellos en la región h con salario superior a la media. En la medida en que las distribuciones de los grupos sean iguales, es decir, que no exista segregación, el indicador se aproximará a 1, mientras que, si las diferencias entre los grupos aumentan, el valor del indicador se aproximará a 0 , indicando la condición máxima de segregación.

Al realizar la simulación se evidenciará la evolución del índice en cada ronda. La realidad que se presenta es un aumento de la segregación entre las dos plazas que es originada por las condiciones diferentes de escogencia de los hogares. De esta manera, se plantea necesario un cambio en la distribución del recaudo impositivo, de tal forma que con el impuesto recogido en la zona desarrollada se financien los bienes públicos de la zona rezagada. Tal aumento de los bienes públicos generará un incremento (en el agregado final) de la utilidad de los hogares de dicha zona, que, en palabras de Sen, puede explicarse como aumento de la calidad de vida. Evidentemente, todavía existirán las 
diferencias de utilidades entre las dos zonas, pero se ha originado un proceso de mejoramiento de las condiciones de vida que posibilita un aumento de capacidades en la región atrasada y, con ello, una mejor distribución del desarrollo en función de las capacidades.

\subsection{Simulación del modelo}

El presente modelo de simulación, utilizando la plataforma Netlogo ${ }^{12}$, permite observar, en primer lugar, la segregación de agentes dadas sus condiciones propias en el hogar y según las condiciones de los lugares donde se ubican, y, en segundo lugar, un proceso de disminución de tal desigualdad a partir de una política distributiva que beneficia la calidad de vida (utilidad) de los rezagados.

\section{Características}

Las características de los hogares vienen dadas por las siguientes variables:

- $\quad$ Salud (s)

- $\quad$ Educación (e)

- $\quad$ Salario $(w)$

- Coeficiente para salud, coeficiente para educación.

Mientras que cada una de las dos regiones se caracteriza por los siguientes aspectos:

- $\quad$ Bien público $(\beta)$

- Costos asociados a la vivienda $\gamma(\mathrm{p})$

- $\operatorname{Impuestos}(\boldsymbol{\Phi})$.

\section{Descripción de la simulación}

Comúnmente, los agentes (hogares) deciden en que región o área habitar. Sus decisiones se basan de acuerdo con las utilidades que le represente cada una de las dos regiones.

Se retoma la función de utilidad expresada anteriormente para cada hogar (1):

$$
\text { Máx. } \mathrm{V}_{\mathrm{h}}^{\mathrm{i}}=\alpha_{\mathrm{x}}^{\mathrm{i}} \mathrm{x}_{\mathrm{h}}^{\mathrm{i}}+\beta_{\mathrm{h}}-\gamma_{\mathrm{p}}^{\mathrm{i}} \mathrm{p}_{\mathrm{h}}-\Phi\left(\mathrm{w}_{\mathrm{h}}^{\mathrm{i}}\right)
$$

Por características del hogar $(\alpha)$ tomamos educación, salud y salario como condiciones proxies del IDH. Es decir, $\alpha(\mathrm{s}, \mathrm{e}, \mathrm{w})$.

12 Por cuestiones de espacio, en el presente texto no se incluye el anexo respectivo de los algoritmos y códigos utilizados que se presentaron en la exposición de la tesis. Para quien desee estudiarlo, me puede escribir, y con gusto lo suministraré. 
La variable del bien común $(\boldsymbol{\beta})$ está determinada por los impuestos $(\boldsymbol{\Phi})$ al salario de cada región.

El precio de la vivienda está determinado por el promedio de salarios de cada región.

El impuesto es un parámetro determinado como el $2 \%$ del salario de cada agente, independientemente de la zona donde se encuentre.

Una vez cada agente calcula su utilidad esperada de acuerdo con cada región, entonces elige estar en la región que le represente mayor utilidad. La elección de en cuál región estar se actualiza en cada momento. De igual manera, los valores tanto de los hogares como de las regiones se actualizan una vez se distribuyen los hogares según sus elecciones. Así, la comparación de las utilidades esperadas va cambiando conforme a las características que varían en el tiempo, y tales características en un tiempo dado dependen de la decisión del tiempo $t-1$.

\subsection{Procedimientos o actividades de los agentes y del ambiente en la simulación}

Existen dos momentos importantes en la simulación. En primer lugar, la escogencia de características tanto de los agentes como del ambiente al iniciar la simulación y, en segundo lugar, las actualizaciones de esas características en cada momento. Tales actualizaciones se originan por la forma como están relacionadas entre sí las variables tanto de los agentes como de las regiones, que en nuestro modelo dependen de la función de utilidad, tal como se presentó en el apartado anterior.

Para el tiempo inicial de la simulación se determinaron los valores de las características de las regiones y la dotación inicial de los agentes (hogares). En el caso de las regiones, se determinaron cuántos agentes existen en él, los valores de los bienes públicos y los valores del precio de la vivienda de cada zona. La escogencia discrecional de los valores iniciales expresa las diferencias de desarrollo entre las dos regiones. Los valores escogidos, como se verá a continuación, son pertinentes en tanto que caracterizan, de entrada, la situación de aglomeración donde una región tiene un nivel de desarrollo más avanzado que la otra, nivel que se evidencia en las características propias de los hogares y de las regiones. En otras palabras, la disparidad de las dos regiones es fruto del proceso de aglomeración que se ha descrito a lo largo de la presente tesis y que Krugman asimila con base en el Modelo Centro-Periferia.

Por el otro lado, los hogares son ubicados de forma aleatoria en alguna de las dos zonas, y a partir de esta relación región-hogares también se definen los valores de salud, educación y salario, que corresponden a las características principales de los hogares. 
En el momento inicial de la simulación, el ambiente es caracterizado con los siguientes valores:

- El bien público inicial de cada área

- Bien público área $0=0.05$

- $\quad$ Bien público área $1=0.06$

- El precio de vivienda inicial de cada área:

- $\quad$ Precio vivienda área $0=0.7$

- Precio vivienda área $1=1$

- El número de agentes como un parámetro de la simulación.

En el momento inicial de la simulación, los agentes se caracterizan de la siguiente manera:

- Se ubican en alguna de las dos áreas: $(\mathrm{h}=\{0,1\})$

- Si el agente se ubica en el área $\mathrm{h}=0$, entonces:

- $\quad$ el coeficiente de salud $(0.98-1)$

- el coeficiente de educación $(0.98-1)$

- Si el agente se ubica en el área $\mathrm{h}=1$, entonces:

- el coeficiente de salud $(1-1.05)$

- el coeficiente de educación $(1-1.05)$

- Se determina el salario de cada hogar de acuerdo con:

- $\quad$ salario $=($ salud + educación $) 2 / 2$

- Por último, cuando los agentes ya están caracterizados, se determinan el bien público y los promedios de los salarios de cada área.

Una vez se tiene el entorno de simulación definido, comienza la primera simulación, que consiste en observar y analizar los comportamientos de los hogares y cómo estos agentes cambian sus características por medio de interacciones entre agentes-agentes y ambiente-agente. Los comportamientos de la simulación corresponden a instrucciones que realiza cada hogar, modificando sus valores tales como salud, educación y salario.

Para los siguientes periodos, y teniendo en cuenta las condiciones iniciales de los hogares y del ambiente (representado en dos áreas), las características se actualizan de la siguiente manera: 
- Se actualizan las características de los hogares, como son la educación, salud y salario.

- Se determinan los impuestos al salario de cada agente.

- Para cada área se determinan el bien público y el promedio de los salarios tomando en cuenta las características de los agentes de cada zona.

- Se actualiza el precio de vivienda de cada área.

Una vez definidas las características, cada hogar proyecta el valor esperado de su utilidad conforme a los valores propios (salud, educación y salario) y los valores de las dos áreas (bien público y vivienda). De esta manera, cada hogar proyecta dos funciones de utilidad y, según el área que le brinde mayor utilidad, decide ubicarse en ella. Así vuelve a empezar el proceso de actualización de características conforme al nuevo ordenamiento de los hogares y, con ello, un nuevo momento de comparación de utilidades esperadas y de decisión.

Lo anterior se describe en la siguiente figura, en la que se establecen los procedimientos o actividades que cada hogar hace y el ambiente en una simulación.

Figura 1. Actividades de los agentes y actividades en las áreas
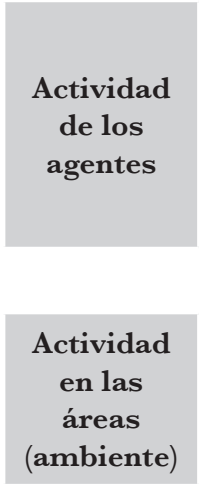

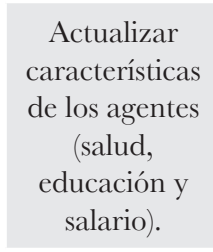

Determinar el bien público de cada área.

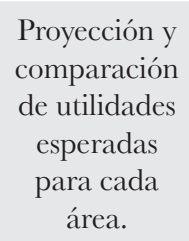
comparación de utilidades esperadas para cada área.

Actualizar precio de vivienda de cada área.

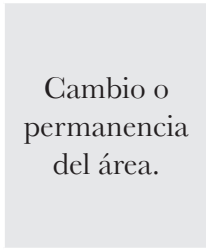

Actualizar características de los agentes (salud, educación y salario).

Fuente: elaboración propia

Desde la ubicación aleatoria de los agentes y la formalización de los procesos y las áreas que recogen las características de un simulacro de una economía en aglomeración, se adelanta la "corrida" del modelo durante un tiempo de 0 a 100 pulsasiones. Sin acciones distributivas, el modelo arroja una disparidad demostrativa entre las zonas, expresada en el índice de segregación que se va calculando al tiempo. A continuación, la proyección origina un proceso de distribución económica que permite propiciar un fortalecimiento de los bienes públicos de la zona rezagada a partir de los impuestos al salario de los hogares de la zona desarrollada. El mejoramiento de los bienes públicos posibilita un aumento de capacidades de aquellos que se benefician de ellos y una reducción de 
la distancia entre el promedio de utilidades de las dos regiones; con ello, el índice de segregación disminuye.

Tabla 2. Paralelo entre el tiempo 100, donde ocurre segregación, y el tiempo 100, donde hay distribución económica.

\begin{tabular}{|c|c|c|c|c|}
\hline & \multicolumn{2}{|c|}{ Tiempo 100 segregación } & \multicolumn{2}{|c|}{ Tiempo 100 distribución } \\
\hline & Área 0 & Área 1 & Área 0 & Área 1 \\
\hline Impuesto $(\Phi)$ sobre el salario & $2 \%$ & $2 \%$ & $2 \%$ & $2 \%$ \\
\hline Bien público $(\beta)$ & $\beta(\Phi 0)$ & $\beta(\Phi$ l) & $\beta(\Phi 0+\Phi 1 / 2)$ & $\beta(\Phi 1 / 2)$ \\
\hline $\begin{array}{l}\text { Diferencia máxima entre IDH } \\
\text { (proxy) de las dos áreas }\end{array}$ & \multicolumn{2}{|c|}{0.66} & \multicolumn{2}{|c|}{0.428} \\
\hline $\begin{array}{l}\text { Diferencia máxima promedio } \\
\text { de utilidades entre las áreas }\end{array}$ & \multicolumn{2}{|c|}{0.14} & \multicolumn{2}{|c|}{0.12} \\
\hline Índice de segregación ${ }^{13}$ & \multicolumn{2}{|c|}{0.446} & \multicolumn{2}{|c|}{0.451} \\
\hline
\end{tabular}

\section{Conclusiones}

La discusión planteada entre Krugman y Sen se ha enmarcado dentro de la tradición teórica de aquellos que han debatido la noción de desarrollo. Desde un principio se reconoció que los dos autores abordan el problema desde ópticas diferentes. Por un lado, Krugman hace su estudio desde una perspectiva histórica-analítica que intenta describir el proceso de desarrollo que han tenido algunos lugares a diferencia de (pero en relación con) otros que han quedado olvidados por la fuerza del progreso. Los textos que abordamos del nobel planteaban una situación de lugares que se comparaban según sus dinámicas económicas. La obviedad de observar lugares desarrollados en contrapartida de lugares no prósperos fue cabalmente descrita por Krugman en su teoría, siendo para nosotros de vital interés el proceso de aglomeración. De la variada riqueza conceptual y del horizonte de problemas que abre un pensador como Krugman, nos centramos justamente en esa diferencia de crecimiento entre las plazas, entre el mundo desarrollado y el mundo rezagado. Apoyándonos un poco en el Informe del Banco Mundial de 2009, pudimos notar que la desigualdad era un producto inevitable del devenir de la fuerza de la aglomeración y las economías de escala, siendo algo entendible y aceptable mientras se posibilitara el proceso paulatino de contagio.

En esta teoría económico-geográfica situamos en debate la vigencia del pensamiento de Amartya Sen sobre el tema de desarrollo, en particular sus propuestas sobre elementos

13 Tal como se mencionó con anterioridad, el índice de segregación varía entre 0 a 1, siendo 0 total segregación y 1 no segregación. 
(factores) de superación de la pobreza y la desigualdad. A la amplia comprensión de los procesos de desarrollo económico de Krugman, la connotación profunda de desarrollo como libertad de Sen permitió abordar los procesos de desarrollo desde una mirada no percibida en el pensamiento de Krugman. Mientras que este último se concentra en las dinámicas de las empresas, Sen se sitúa en la posición de las personas, de sus condiciones reales y la forma como se puede mejorar su calidad de vida.

La propuesta de Sen de desarrollo como aumento de capacidades irrumpe en la teoría de Krugman para cuestionarla sobre los alcances de su concepción de desarrollo, que no pretende dar respuesta cabal a las zonas atrasadas. De tal cuestionamiento surge la necesidad de pensar el proceso de desarrollo desde la aglomeración y los centros de producción a partir de acciones que promuevan y conlleven desarrollo humano a las capas de la sociedad no favorecidas por tal proceso de aglomeración. Con el presente trabajo auspiciamos no una contradicción entre la nueva geografía económica y el desarrollo humano, sino una posibilidad de complemento y conjunción en la que se reconozca el devenir de las empresas en su búsqueda de maximizar sus beneficios, pero ligado a una eventual propuesta afirmativa desde la política económica que potencie las capacidades de los que no gozan ese ordenamiento económico. De esta manera, se abre la posibilidad de mirar mecanismos de distribución donde las zonas más desarrolladas contribuyan al mejoramiento de la calidad de vida de las zonas rezagadas. No se trata de interrumpir el proceso de las economías de aglomeración, sino de reconocer sus deficiencias y efectos negativos, y de brindar instrumentos para, en lo posible, generar un proceso de desarrollo más equitativo y humano.

Mediante la simulación lograda en la plataforma Netlogo se pudo avizorar una posible reducción de la desigualdad entre dos lugares con características económicas dispares. Tal reducción se pudo conseguir por medio de una contribución de los agentes favorecidos que, mediante una proporción de sus impuestos, financian bienes públicos de la región segregada. Los bienes públicos generan impactos positivos en las condiciones (capacidades) de los agentes de dicha zona y posibilitan el aumento de sus utilidades o, en sentido amplio, de su calidad de vida. El fortalecimiento de tales bienes púbicos es una estrategia política de distribución de oportunidades que impulsa la reducción de la desigualdad. Evidentemente, se tendrá una certeza de la idoneidad del modelo una vez se pueda desarrollar posteriormente con datos reales.

Este trabajo es un acercamiento que permite proyectar un interesante campo de trabajo que tendrá que incluir con mayor cabalidad fuerzas económicas que no se tomaron en cuenta por conveniencia estructural y teórica del modelo, por ejemplo, los costos asociados al transporte, que son de gran importancia para la nueva geografía económica y que, como experiencia de investigación, fueron incorporados hábilmente en el "índice de desarrollo humano urbano" (IDHU) del Informe del Desarrollo Humano para Bogotá de 2008. 


\section{Referencias}

Banco Mundial. (2009). Informe sobre el desarrollo mundial. Una nueva geografia económica (Trad. I. Caviedes Hoyos). Colombia: Banco Mundial, Mundi-Prensa y Mayol Ediciones S. A. Recuperado de http://goo.gl/fMJU9W

Fujita, M., \& Krugman, P. (2004). La nueva geografía económica: pasado, presente y futuro. Investigaciones regionales, 4, 117-206. Recuperado de http://www.redalyc.org/ articulo.oa?id=28900409

Henderson, J., Shalizi, Z., \& Venables, A. (2001). Geography and development. fournal of Economic Geography, 1(1), 81-105.

Krugman, P. (1993). Geografia y comercio. Barcelona: Plaza y Janés. . (1997). Desarrollo, geografia y teoría económica. Barcelona: Antoni Bosch Editor. . (1998). The role of geography in development. Documento preparado para la Conferencia Anual del Banco Mundial sobre Economía del Desarrollo, Washington. PNUD. (2008). Bogotá. Una apuesta por Colombia. Informe de desarrollo humano 2008. Bogotá: PNUD, IDH. Recuperado de http://goo.gl/xbtGfE

Reyes Morris, V. (2002). Cuadernos de trabajo n. 24. La medición de la pobreza y el bienestar y el pensamiento de Amartya Sen. Bogotá: Universidad Nacional de Colombia.

Sen, A. (1997). Bienestar, justicia y mercado. Barcelona: Ediciones Paidós. . (2000). Desarrollo y libertad. Bogotá: Planeta. - (2001). La desigualdad económica. México: Fondo de Cultura Económica

Sen, A., \& Anand, S. (2000). Human development and economic sustainability. World Development, 28(12), 2029-2049.

Vélez, J. (2008). Los procesos de aglomeración en Colombia a la luz de la nueva geografía económica. Ensayos sobre Política Económica, 27(58), 106-139. 